\title{
Clinical telemedicine utilization in paediatric nephrology at a tertiary care centre
}

\author{
Fei Fei Shao, Jody Andody, April Reed, Guido Filler
}

Faculty Reviewer: Tim Carey, MD, FRCSC (Department of Paediatric Orthopedic Surgery), Debra Bartley, MD, FRCSC (Department of Paediatric Orthopedic Surgery)

\section{ABSTRACT}

Purpose: Telemedicine is an emerging tool that offers medical consultations to patients with limited access to subspecialty care. To date, the use of telemedicine in Canadian medical practices has not been studied in depth. This study aims to characterize the three different telemedicine clinic models in use at the paediatric nephrology program at the Children's Hospital of Western Ontario and the patient population they served.

Methods: To complete this retrospective study, all paediatric nephrology telemedicine consultations provided by one physician from September 30, 2010 to July 13, 2016 were analyzed, which comprised 264 separate consultations with 87 patients.

Results: The number of consults increased from 0.25 per month to 8.78 per month between 2010 and 2016, with the most substantial increase seen from 2014 onwards with the establishment of the Windsor block clinics. The patient population had a bimodal age distribution across a geographical distance of 220.3 $\mathrm{km}$ to $1371.31 \mathrm{~km}$. Urinary tract infections were the most frequent reason for the consultation.

Conclusion: Of the three models described in this study, the block clinic model had the most substantial impact on increasing telemedicine services for paediatric nephrology patients.

\section{INTRODUCTION}

Telemedicine is becoming an important tool for providing patients with medical care. ${ }^{1}$ In paediatrics alone, there has been an increase in the use of telemedicine for mental health, ${ }^{2}$ diabetes, ${ }^{3}$ and respirology. ${ }^{4}$ We are only aware of one study that has looked at the use of telemedicine in paediatric nephrology, which was conducted in Brisbane by Trnka et al. ${ }^{5}$ They found telemedicine to be a viable and economic method for patient assessment and follow-up. The paediatric nephrology catchment area for Western University is very large and serves over 690000 children located from Southeastern Ontario up to the Manitoba border. ${ }^{6}$ Sparsely populated areas such as Northern Ontario with approximately 775000 people scattered across an area of $803000 \mathrm{~km}$ stand to benefit most from improved subspecialty care access through telemedicine, though all patients with transportation or mobility limitations would benefit from its use. ${ }^{2,7}$ So far, physicians have had a relatively low interest in telemedicine due to concerns over inefficiencies of scheduling in the form of stand-alone appointments outside of regular clinic hours. ${ }^{8,9}$ In this retrospective study, we used medical records collected between September 30, 2010 and July 13, 2016 inclusively from a single physician in the paediatric nephrology department at
Western (author GF). Telemedicine consultations were delivered with secure video-feed provided through the Ontario Telemedicine Network (OTN) and through three different clinic models:

1. Stand-alone consultations with no other sessions booked prior to or following this single consultation. A single OTN connection is created between the physician video-feed station and the video-feed station accessed by the patient.

2. Time blocks of consecutive 30 minute telemedicine consultations, organized by the physician's secretary (author JA), where a single physician telemedicine station connects to multiple remote-access stations. New OTN connections would be established for each consultation.

3. "Block Clinic Model" - A continuous 4 hour video-feed is secured between a single physician video-feed station and a single video-feed station accessed by patients. This telemedicine clinic model mimics the structure of a typical out-patient clinic with patients seen in consecutive 30 minute consultations. In the summer of 2014, a monthly "block clinic" was established within the Windsor Regional Hospital (WRH) - Metropolitan Campus.

Studies examining the use of telemedicine in paediatric nephrology are scarce and published literature on telemedicine use has not specifically examined the block clinic model.,10 This study aims to review the nature of telemedicine activity and analyze the impact of the block clinic model on telemedicine use at Western's paediatric nephrology program.

\section{METHODS}

\section{Clinic Model and Setting:}

The study was conducted at Western University and the Children's Hospital of Western Ontario (CHWO), which is a part of London Health Sciences Centre. The Children's Hospital is a medium-sized paediatric academic health sciences center with a catchment area of 690000 children from four Local Health Integrated Networks (LHINs) in Ontario, namely the Erie St. Clair, South West, Waterloo Wellington, and North West LHINs, the latter of which stretches from Sault St. Marie to the Manitoba border. The "Block Clinic" at WRH is structured similarly to a typical hospital out-patient clinic. Patients arrive 15 minutes prior to their telemedicine consultation and a Windsor nurse practitioner (author AR) would obtain anthropometry and perform a physical and a medication reconciliation. At the beginning of each consult, the nurse would report these medical information to the physician then leave to examine the next patient while the physician interacted with the patient. A single block clinic has served up to 7 consecutive patients. 


\section{Data Collection:}

The nephrology program established a telemedicine database in September 2010. The study was exempt from Research Ethics Approval because it only used a consult number and location, rather than individual patient data. The database included remote-access station locations, consult dates, patient codes, birth dates, and primary diagnoses. The distance from the hospital to the telemedicine station was calculated using a distance calculator (www.distancecalculator.globefeed.com) and was expressed as air distance. The age of the patient at his or her first visit was calculated by subtracting his or her birth date from the consult date.

\section{Statistical Analysis:}

Simple descriptive statistics were used whenever possible. Data was assessed for normality using the Kolmogorov-Smirnov test. Normally distributed data was summarized by mean and standard deviation, while skewed data was summarized by median and interquartile range (IQR). The spectrum of diagnoses was organized into contingency tables and analyzed using Chi-squared tests. The statistical analysis and the graphs were generated using GraphPad Prism 5 Version 5.0.

\section{RESULTS}

\section{Patient Population:}

A total of 264 consultations with 87 patients took place during the study period, with only 3 new consultations. Telemedicine was primarily used for follow-ups (including antenatal counseling). The mean patient age was $9.0 \pm 6.4$ years and the median age was 7.7 years (minimum 0.1 years, 25 th percentile 3.8 years, 75 th percentile 14.1 years and maximum 31.5 years). A frequency distribution analysis of the patients' age at their initial visit indicates two peaks, one at pre-school age and one at adolescence (Figure 1). The consults took place at 33 different locations (Figure 2). St. Thomas Elgin Hospital was the closest location $(22.03 \mathrm{~km})$, and Pikangikum $(1371.31 \mathrm{~km})$ and Summer Beaver $(1218.34 \mathrm{~km})$ were the two furthest locations. WRH, Metropolitan Campus (162.46 km) saw the highest number of consults. Table 1 summarizes the statistics of each remote-access station.

\section{Diagnoses:}

We compared the diagnoses of our telemedicine patients ( $\mathrm{Ta}-$ ble 2) with the results of Trnka et al. ${ }^{5}$ The spectrum of diagnoses were significantly different between these two studies (Chi-square test $\mathrm{p}<0.0001)$. The Brisbane group encountered fewer patients with hypertension and fewer patients with urinary tract infections but a greater total number of consults.

\section{Telemedicine Activities:}

The frequency of telemedicine consults increased yearly from 0.25 per month to 8.78 per month between 2010 and 2016 (Figure 3 ). The large number of telemedicine consults conducted at the Windsor site following the introduction of the block clinic model, contributed to the substantial increase in the total number of telemedicine consultations from 2014 onwards (Figure 3).
Table 1. Number of consults, patients, and air distance from Western University by telemedicine station. Data was not normally distributed and summarized by median and interquartile range (IQR).

\begin{tabular}{|c|c|c|c|}
\hline Location & No. consults & No. of patients & $\begin{array}{l}\text { Distance from } \\
\text { center }(\mathrm{km})\end{array}$ \\
\hline Windsor & 96 & 34 & 184.1 \\
\hline Sturgeon Falls & 33 & 2 & 392.3 \\
\hline Sudbury & 25 & 4 & 390.3 \\
\hline Listowel & 16 & 5 & 88.8 \\
\hline Leamington & 12 & 3 & 152.4 \\
\hline Thunder Bay & 12 & 3 & 866.2 \\
\hline Fort Hope & 11 & 2 & 1079.4 \\
\hline Chatham & 7 & 5 & 101.5 \\
\hline Walkerton & 6 & 1 & 130.4 \\
\hline Kincardine & 5 & 1 & 139.1 \\
\hline Cambridge & 4 & 2 & 86.3 \\
\hline Seaforth & 4 & 2 & 70.0 \\
\hline Clifford & 3 & 1 & 114.0 \\
\hline St. Thomas & 3 & 1 & 20.5 \\
\hline Hanover & 2 & 1 & 133.6 \\
\hline Kitchener & 2 & 1 & 81.1 \\
\hline Meaford & 2 & 2 & 190.0 \\
\hline Owen Sound & 2 & 1 & 180.4 \\
\hline Sarnia & 2 & 1 & 96.1 \\
\hline Stratford & 2 & 1 & 49.6 \\
\hline Wellsley & 2 & 1 & 73.7 \\
\hline Barrie & 1 & 1 & 201.2 \\
\hline Brantford & 1 & 1 & 80.0 \\
\hline Goderich & 1 & 1 & 95.6 \\
\hline Grand Bend & 1 & 1 & 58.3 \\
\hline Harrow & 1 & 1 & 172.7 \\
\hline Ingersol & 1 & 1 & 29.1 \\
\hline Kingston & 1 & 1 & 406.9 \\
\hline Markdale & 1 & 1 & 158.0 \\
\hline Pikangikum & 1 & 1 & 1371.3 \\
\hline Strathroy & 1 & 1 & 32.4 \\
\hline Summer Beaver & 1 & 1 & 1214.8 \\
\hline Toronto & 1 & 1 & 167.7 \\
\hline West Lorne & 1 & 1 & 50.4 \\
\hline Total & 264 & 87 & $\begin{array}{l}\text { Median } 132 \text { km } \\
(\text { IQR 78.4, 192.8) }\end{array}$ \\
\hline
\end{tabular}

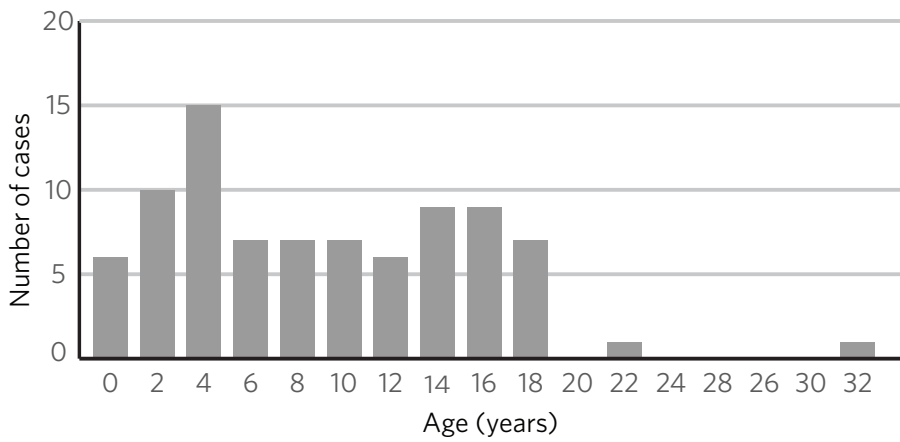

Figure 1. Frequency distribution of patient age at their first telemedicine consultation. Age distribution is biphasic with two modes, one at pre-school age and one at adolescence.

\section{DISCUSSION}

The telemedicine "block clinic" model established in WRH, Metropolitan Campus substantially contributed to the growth in number of total telemedicine consults. Since its establishment in 2014, the rapid increase in consultations conducted from this remote-access telemedicine station is suggestive of this clinic model's 


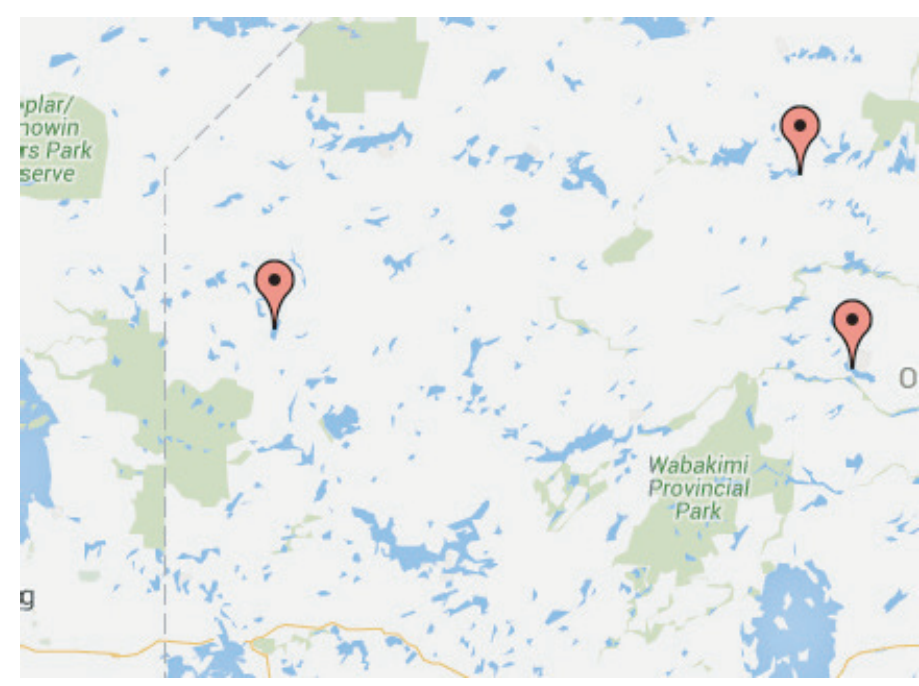

Figure 2. Map of the remote-access telemedicine stations used over the study period, generated using Google Maps (https://www.google.ca/maps).

Table 2. Paediatric nephrology consultations by the primary diagnosis of patients.

\begin{tabular}{lcc}
\hline Spectrum of diagnoses & No. of patients & Percent \\
\hline CAKUT & 16 & $18.4 \%$ \\
Nephrotic Syndrome & 15 & $17.2 \%$ \\
Hypertension & 10 & $11.5 \%$ \\
CKD & 8 & $9.2 \%$ \\
AKI & 6 & $6.9 \%$ \\
Glomerulonephritis & 6 & $6.9 \%$ \\
Stones/hypercalciuria & 5 & $5.7 \%$ \\
Transplant & 3 & $3.4 \%$ \\
Diabetic nephropathy & 3 & $3.4 \%$ \\
Voiding Dysfunction & 3 & $3.4 \%$ \\
Recurrent UTI & 3 & $3.4 \%$ \\
Antenatal consult & 1 & $1.1 \%$ \\
Other & 8 & $9.2 \%$ \\
\hline Total & 87 & $100 \%$ \\
\hline
\end{tabular}

CAKUT - congenital anomalies of the kidney and urinary tract; CKD - chronic kidney disease $\mathrm{AKI}$ - acute kidney injury; UTI - urinary tract infection.

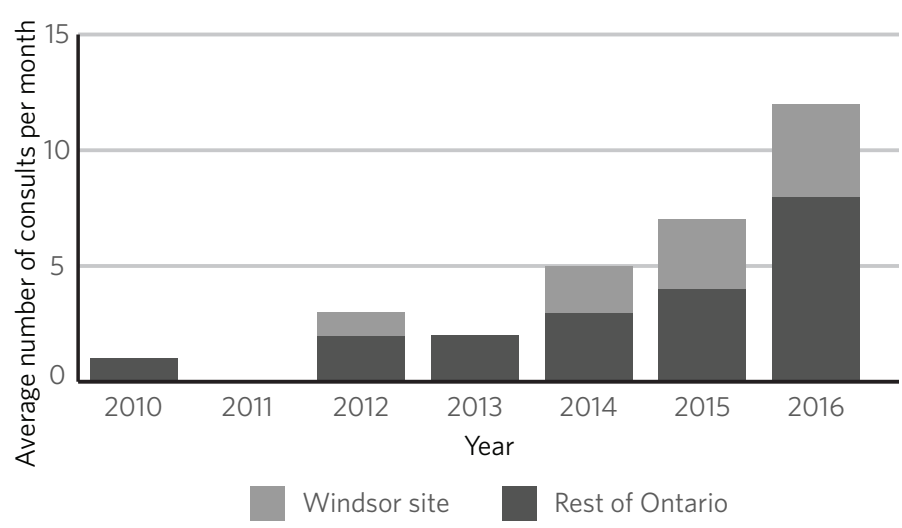

Figure 3. Frequency of total telemedicine consultations per month over the study period, comparing the Windsor Block Clinic (white) versus the rest of Ontario (black). viability and capacity.

Our study had a significantly higher proportion of patient follow-up to new consults (261/264) than the study described by Trnka et al. (165/318). ${ }^{5}$ This pattern can be attributed to the voiced preferences of patients, who preferred in-person initial consults with subsequent telemedicine follow-ups, complemented by yearly in-person consultations. The higher proportion of new consultations in Trnka's study may have also influenced the spectrum of diagnoses, leading to more consultations related to conditions that are typically seen only once (eg dysfunctional voiding). For both studies, the majority of telemedicine consultations involve patients with sub-acute or chronic kidney problems who do not require complex work-ups or specialized hospital equipment. While congenital anomalies of the kidney and urinary tract (CAKUT) still comprised a major portion of primary patient diagnoses (18.4\%) in telemedicine consultations, this was less frequent than typically observed with in-hospital diagnoses, possibly because these patients require a paediatric ultrasound of the kidneys and urinary tract, which is difficult to coordinate remotely. ${ }^{11}$

This study is limited by the source of the data, which was collected from the telemedicine activity of a single physician. The interests and practicing patterns of the physician influence the patients' ages, patient diagnoses, remote-access sites, and consultation frequencies. The results may have also been confounded by the population served in the relatively large city of Windsor, Ontario, and the quality of support staff at the telemedicine site. These enabling factors vary widely throughout the catchment area.

In summary, the use of telemedicine-based consultations in the paediatric nephrology program at CHWO has been growing, particularly with the introduction of the Windsor block clinics. In a country like Canada, with a large but sparsely-populated geographical area, telemedicine can play an important role in reducing travel costs and improving the access of rural and remote-living patients to specialist services. Studies have shown that the complementary use of telemedicine and in-person consultations is a viable and economic method of medical practice that does not result in a decrease in the quality of care, ${ }^{12,13}$ and may improve the quality of care for renal patients. ${ }^{14}$ Of the three models described in this study, the block clinic model established in Windsor had the most substantial impact on increasing the number of telemedicine consultations. This clinic model, first described in this study, provides a good template for future nephrology and non-nephrology paediatric block clinics that could be set-up in similar regional sites such as Sudbury or Thunder Bay. In view of the significant time and costs that are saved by patients and parents by employing telemedicine, the widespread implementation of such models will help to increase the use of telemedicine as a whole.

\section{ACKNOWLEDGEMENTS}

We would like to thank Marta Kobrzynski, Research Assistant to GF, for editing the manuscript. 


\section{REFERENCES}

1. Flewelling C, Ingram CA. Telepediatrics in Canada: an overview. Telemedicine journal and e-health : the official journal of the American Telemedicine Association. 2004;10(3):357-68.

2. Lingley-Pottie P, McGrath PJ. Telehealth: a child and family-friendly approach to mental health-care reform. Journal of telemedicine and telecare. 2008;14(5):225-6.

3. Shulman RM, O'Gorman CS, Palmert MR. The impact of telemedicine interventions involving routine transmission of blood glucose data with clinician feedback on metabolic control in youth with type 1 diabetes: a systematic review and meta-analysis. International journal of pediatric endocrinology. 2010;2010.

4. Morrison D, Wyke S, Agur K, et al. Digital asthma self-management interventions: a systematic review. Journal of medical Internet research. 2014;16(2):e51.

5. Trnka P, White MM, Renton WD, et al. A retrospective review of telehealth services for children referred to a paediatric nephrologist. BMC nephrology. 2015;16:125.

6. Radina M, Sharma AP, Yasin A, et al. Adherence to waiting-time targets for pediatric nephrology clinic referrals. Pediatr Nephrol. 2010;25(2):311-6.

7. O'Gorman LD, Hogenbirk JC, Warry W. Clinical Telemedicine Utilization in Ontario over the Ontario Telemedicine Network. Telemedicine journal and e-health : the official journal of the American Telemedicine Association. 2016;22(6):473-9.

8. Barton PL, Brega AG, Devore PA, et al. Specialist physicians' knowledge and beliefs about telemedicine: a comparison of users and nonusers of the technology. Telemedicine journal and e-health : the official journal of the American Telemedicine Association. 2007;13(5):487-99.

9. Grigsby B, Brega AG, Bennett RE, et al. The slow pace of interactive video telemedicine adoption: the perspective of telemedicine program administrators on physician participation. Telemedicine journal and e-health : the official journal of the American Telemedicine Association. 2007;13(6):645-56.

10. Braverman J, Samsonov DV. A study of online consultations for paediatric renal patients in Russia. Journal of telemedicine and telecare. 2011;17(2):99-104.

11. Filler G, Payne RP, Orrbine E, et al. Changing trends in the referral patterns of pediatric nephrology patients. Pediatr Nephrol. 2005;20(5):603-8.

12. Gomez-Martino JR, Suarez MA, Gallego SD, et al. [Telemedicine applied to Nephrology. Another form of consultation]. Nefrologia. 2008;28(4):407-12.

13. Long R, Heffernan C, Gao Z, et al. Do "Virtual" and "Outpatient” Public Health Tuberculosis Clinics Perform Equally Well? A Program-Wide Evaluation in Alberta, Canada. PloS one. 2015;10(12):e0144784.

14. Norum J, Leivestad T, Eriksen BO, et al. Regional differences in renal replacement therapy in northern Norway 2000-2012. International journal of circumpolar health. 2015;74:24298.

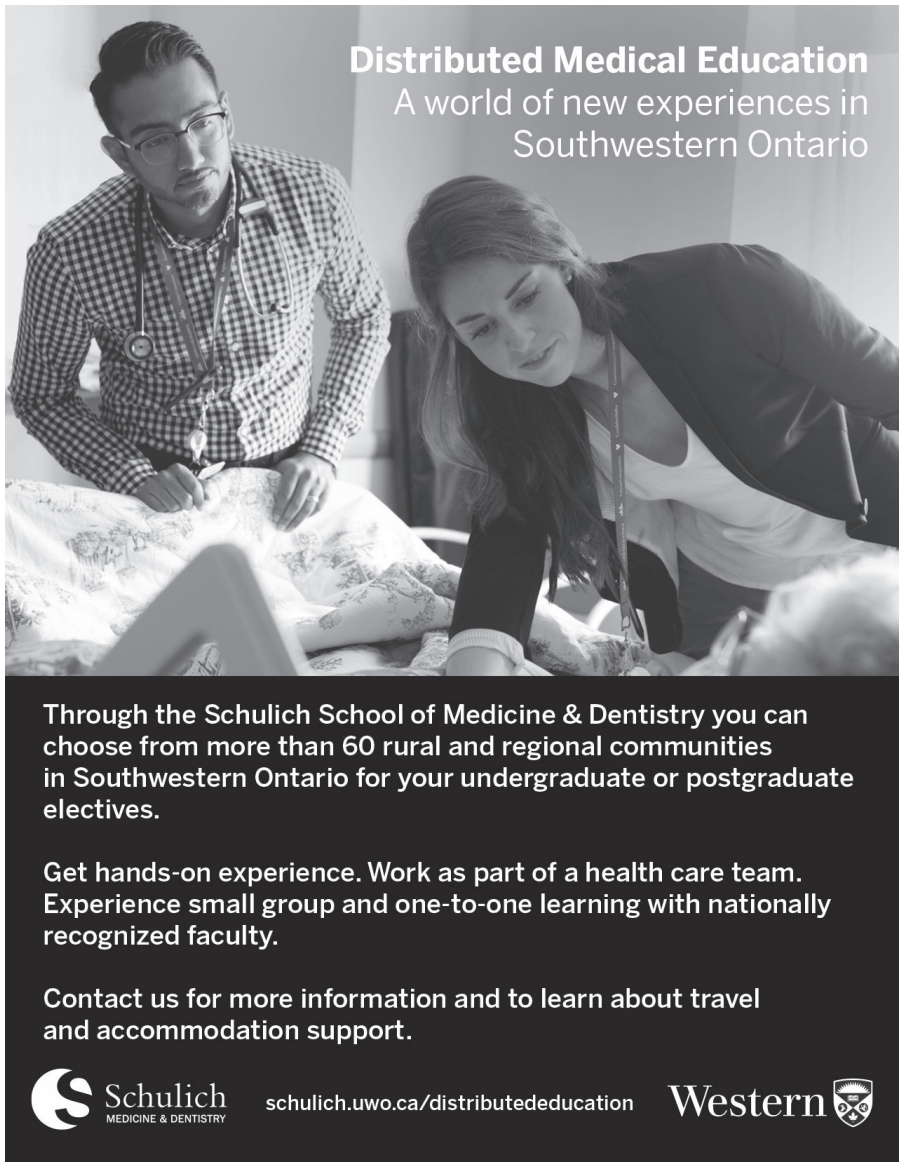
Southwestern Ontario for your undergraduate or postgraduate Experience small group and one-to-one learning with nationally

Contact us for more information and to learn about trave and accommodation support. 